\title{
1 Facilitating Replication and Reproducibility in Team Science: The 'projects' R Package
}

3 Nikolas I. Krieger ${ }^{1}$, Adam T. Perzynski ${ }^{2}$, and Jarrod E. Dalton ${ }^{1,3}$

4

$5 \quad{ }^{1}$ Department of Quantitative Health Sciences, Lerner Research Institute, Cleveland Clinic,

6 Cleveland, Ohio, United States of America

7

$8 \quad{ }^{2}$ Center for Healthcare Research and Policy, Case Western Reserve University at

9 MetroHealth, Cleveland, Ohio, United States of America

10

${ }^{3}$ Cleveland Clinic Lerner College of Medicine, Case Western Reserve University, Cleveland,

12 Ohio, United States of America

13

14

$15 *$ Corresponding author

16 E-mail: kriegen@ccf.org (NIK) 


\section{Abstract}

19 The contemporary scientific community places a growing emphasis on the reproducibility

20 of research. The projects R package is a free, open-source endeavor created in the interest

21 of facilitating reproducible research workflows. It adds to existing software tools for

22 reproducible research and introduces several practical features that are helpful for

23 scientists and their collaborative research teams. For each individual project, it supplies an

24 intuitive framework for storing raw and cleaned study data sets, and provides script

25 templates for protocol creation, data cleaning, data analysis and manuscript development.

26 Internal databases of project and author information are generated and displayed, and

27 manuscript title pages containing author lists and their affiliations are automatically

28 generated from the internal database. File management tools allow teams to organize

29 multiple projects. When used on a shared file system, multiple researchers can

30 harmoniously contribute to the same project in a less punctuated manner, reducing the

31 frequency of misunderstandings and the need for status updates.

\section{Introduction}

33 The past few years have yielded much discussion and controversy in scientific circles

34 surrounding the so-called replication crisis, in which results of important studies may fail

35 to generalize to other samples. $(1,2)$ Correspondingly, reproducibility and replication are

36 increasingly central goals of the contemporary scientific process. Journals are increasingly

37 emphasizing replicability into account when scrutinizing submissions, with some

38 establishing replication standards for submitted articles. (3) 
39 Reproducibility and replication are related but distinct. The reproducibility (or internal

40 reproducibility) of a study is the ability of its readers to follow its methods and workflow

41 that the researchers used to generate their reported result from their study data. Actively

42 maintaining and archiving this workflow is important to the evaluation of the research: a

43 study is internally reproducible when other researchers can follow the same workflow to

44 achieve the same results from the same data. The external reproducibility of a study is the

45 extent to which its methods and workflow can be meaningfully applied to external data

46 (i.e., data that are different from the original study data). In turn, researchers who

47 externally reproduce the same workflow to achieve similar results to those of the original

48 study using different data provide evidence of replicability. (4) Thus, a study can be both

49 internally and externally reproducible but not replicable if external reproduction attempts

50 fail to produce results similar to those of the original study.

51 There exist today widely available tools that aid with reproducible research, such as $\mathrm{R}$ and

52 other statistical programming languages, that allow for precise documentation of some of

53 the most detail-oriented portions of a project workflow. Researchers can distribute their

54 code scripts alongside their results in order to communicate the integrity of their data

55 processing and analysis. Unfortunately, statistical programming languages per se only

56 contribute to research reproducibility insofar as individual statistical programmers are

57 able (i) to use these tools effectively and (ii) to integrate their own use of these tools with

58 their collaborators' work-which may not necessarily be oriented towards reproducibility.

59 The goal of the projects $\mathrm{R}$ package is to provide a set of tools that support an efficient

60 project management workflow for statisticians and data scientists who perform 
61 reproducible research within team science environments. The projects package is built

62 upon some existing tools for reproducible research, particularly RStudio, the R integrated

63 development environment in which it dwells, and R Markdown, the file structure that

64 allows users to assemble datasets, to perform analyses and to write manuscripts in a single

65 file. The projects package is oriented towards efficient and reproducible academic

66 research manuscript development and incorporates protocol and analysis templates based

67 on widely-accepted reporting guidelines (viz., CONSORT and STROBE). When used on a

68 shared file system (e.g., a server), the projects package provides infrastructure for

69 collaborative research: multiple researchers can work on the same project and keep track

70 of its progress without having to request updates.

71 The primary features of the projects R package are the following:

72 - Relational database containing details of projects, project coauthors and their

73 affiliations, so that author details generally need to be entered only once;

74 - Tools for editing metadata associated with projects, authors and affiliations;

75 - Automated file structure supporting reproducible research workflow;

76 - Report templates that automatically generate title page headers, including a numbered

77 author list and corresponding affiliations;

78 - Full RStudio integration via R Markdown, including customizable styling via cascading

79 style sheets (CSS);

80 - Customization, including the ability to add and to edit templates for protocols and

81 reports, and the ability to change default project directory and file structures; and 
82 - Organization and management functionality, including the ability to group, archive and

83 delete projects.

84 At its outset, the projects package creates a folder called /projects in a user-specified

85 location. This directory will contain all research projects created and maintained by the

86 user. The /projects folder will also contain a relational database of the research projects

87 and the persons who contribute to them. The database allows for users to input important

88 metadata about the projects and authors, including stage of research and contact

89 information. Once this higher-level folder is created, users run R functions to create

90 projects, each of which is given its own folder. New project folders automatically include

91 aptly named subfolders and templates in order to guide the project workflow (e.g., a "data"

92 subfolder; a "datawork" R Markdown template). Henceforth, users can begin working on

93 the research project and edit as needed the metadata of the project itself and its authors. To

94 lessen the burden of the mundane details of manuscript writing, the projects package can

95 output lines to the console that, when copied into an R Markdown file, generates a title

96 page with all relevant authorship information of any given project. Finally, since users may

97 create dozens of projects over time, users can run functions to organize their projects

98 within grouping subfolders of the main / projects folder.

\section{Conceptual Framework: Reproducible Research Workflows}

100 Although researchers of different disciplines may operate in nuanced ways, there are

101 aspects of the project workflow that are common to most investigations. First, studies are

102 conceptualized and designed according to a protocol that details the research questions

103 and planned analyses. Data are collected, manipulated (or "tidied") in order to make data 
104 analysis possible. The results of the analyses are compiled into a report, and ultimately an

105 academic manuscript is drafted and submitted for wider distribution.

106 When navigating this workflow, researchers strive for reproducibility wherever possible

107 but especially during the intermediate, data-focused phases of the workflow. While

108 primary data sources for research projects can be complex, dynamic and diverse, a

109 reproducible analytic workflow ultimately should incorporate a "frozen" dataset that

110 reflects a given set of queries or data collection forms at a specific point in time. A frozen

111 dataset represents the study data's earliest state of simultaneous digitization and

112 consolidation and is almost invariably a digital file or set of files that standard data analysis

113 software can process (e.g., a comma-separated values, CSV, file; a series of CSV files; a

114 compressed-format dataset in the researcher's analytic programming language of choice).

115 From this point in the workflow through the reporting stage, total reproducibility is

116 expected (see Fig 1). Thanks to modern data analysis via statistical programming

117 languages, a reader should be able to exactly reproduce all data-derived results from the

118 frozen dataset alone. Under the reproducible research framework, scientists should

119 operate under the assumption that another scientist with access to the exact scripts the

120 researchers used to produce their results should be able not only to regenerate the study

121 results but to scrutinize every component of the analysis, beginning with data cleaning

122 operations performed on the frozen dataset.

\section{3 [Insert Fig 1]}

124 Fig 1: The entire workflow of manuscript creation with the reproducible portions 125 encircled. 
126 The middle stage of the assumed study workflow can be performed with near perfect

127 reproducibility, but the beginning and ending stages may not. Researchers cannot

128 document every thought process, literature probe and informal conversation that

129 contributes to the development of the initial study protocol, but they should strive to

130 document it as meticulously as possible. Databases tend to be dynamic such that a given

131 analytic dataset is merely a snapshot in time. As for the final stages of project development,

132 journals require that manuscripts adhere to specific and unique stylistic guidelines and

133 that they be digitally submitted with file types that are not independently conducive to

134 reproducibility (e.g., .pdf). For instance, even as RStudio supports the creation of

135 submission-ready documents directly from frozen datasets, the vast majority of project

136 teams include experts who do not use RStudio; therefore, the collaborative manuscript

137 editing process ultimately takes place in an environment (e.g., Microsoft Word) that only

138 supports total reproducibility with extraordinary effort. In light of these realities, during

139 manuscript creation researchers must do their best, keeping the process in reproducible

140 environments for as long as possible and otherwise documenting significant changes and

141 alterations.

\section{2 projects R package}

\section{Installation}

144 The projects R package can be installed with:

145 install.packages("projects") 


\section{Initial Setup}

147 All projects that the user creates with the projects package-as well as its infrastructure-

148 reside in a main folder called /projects. Users need not manually create this directory, and

149 in fact they are encouraged not to manually manipulate any folders that the projects

150 package involves. Instead, users run the function setup_projects( ), providing the full file

151 path of the directory in which the user wants the /projects folder to reside.

\section{Interactive Metadata}

153 Data about authors, institutional and/or department affiliations and projects are stored in

154.rds files within the main / projects directory, so that the user only needs to enter these

155 details once (unless, for example, a co-author changes their name or affiliations). These

156 data are also used to assemble title pages of reports, with automatically generated author

157 lists and lists of author affiliations. We provide a complete example of this process below in

158 the Demonstration section.

159 The main metadata tables that the user interacts with are projects(), authors( ) and

160 affiliations( ), accessible via functions thusly named. The contents of these tables are

161 described in Tables 1-3. Two additional tables are internally created to keep track of

162 associations between authors and projects and between authors and affiliations (see

163 Internal Metadata Tables).

164 Table 1. The projects() Metadata Table

Column

Description 
id

title

short_title

current_owner work on the project may proceed further.

status

deadline_type field, deadline.

deadline

A date indicating some kind of deadline whose meaning is described in the previous field, deadline_type.

An identification number, specifically an integer, unique among the other projects. This number can be used whenever needing to identify this project within projects package functions.

The title of the project. A nonambiguous substring of title (i.e., a substring that does not match any other project) can be used whenever needing to identify this project within projects package functions. This value is also printed in the YAML header of the "protocol" and "report" .Rmd templates that are automatically generated upon project creation. An optional, unique nickname for the project. A nonambiguous substring of short_title (i.e., a substring that does not match any other project) can be used whenever needing to identify this project within projects package functions. This is useful if users cannot remember the long, formal project title nor the project id.

The id of the author who is responsible for taking action in order that A short description of the status of the project. For example, it may elaborate on the value of current_owner and/or stage.

A simple description of the meaning of the date contained in the next 
stage One of six predefined stages of project development that the project is currently in: c("1: design", "2: data collection", "3: analysis", "4: manuscript", "5: under review", "6: accepted").

path The full file path where the project folder is located.

corresp_auth The id of the author who should be contacted for any correspondence relating to the project. This author's name will be specially marked on automatically generated title pages for this project, and his or her contact information will be specially displayed there as well in a “Corresponding Author" section.

creator The id of the author who initially created the project, or the value of Sys.info() ["user"] if the author who ran new_project() did not enter a value.

\section{Table 2. The authors() Metadata Table}

\section{Column Description}

id

An identification number, specifically an integer, unique among the other authors. This number can be used whenever needing to identify this author within projects package functions.

given_names The given name or names of the author. A nonambiguous substring of given_names (i.e., a substring that does not match any other author) can be used whenever needing to identify this author within projects 
last_name The last name or names of the author. A nonambiguous substring of last_name (i.e., a substring that does not match any other author) can be used whenever needing to identify this author within projects package functions. This is included after given_names in the automatically generated title pages of the projects associated with this author.

title The job title of the author.

degree The abbreviation(s) of the author's academic degree(s). This is included after last_name in the automatically generated title pages of the projects associated with this author.

email The email address of the author. This is included in the "Corresponding Author" section of the automatically generated title pages of projects whose corresp_auth field contains this author.

phone The phone number of the author. This is included in the "Corresponding Author" section of the automatically generated title pages of projects whose corresp_auth field contains this author.

\section{Table 3. The affiliations() Metadata Table} Column Description other affiliations. This number can be used whenever needing to identify this affiliation within projects package functions. 
department_name The department name of the affiliation. A nonambiguous substring of department_name (i.e., a substring that does not match any other affiliation) can be used whenever needing to identify this affiliation within projects package functions. This is included in the affiliations section of the automatically generated title page of projects associated with authors with this affiliation.

institution_name The name of the overall institution of the affiliation. A nonambiguous substring of institution_name (i.e., a substring that does not match any other affiliation) can be used whenever needing to identify this affiliation within projects package functions. This is included after department_name in the affiliations section of the automatically generated title page of projects associated with authors with this affiliation.

address The address of the affiliation. This is included after institution_name in the affiliations section of the automatically generated title page of projects associated with authors with this affiliation. It is also included in the "Corresponding Author" section of the title page when a project's corresponding author has this affiliation as his or her primary (i.e., first) affiliation (see Internal

\section{Metadata Tables).}




\section{Internal Metadata Tables}

168 In keeping with relational database theory, there are two .rds files that keep track of the

169 many-to-many relationships between projects and authors and between authors and

170 affiliations. Each has two columns, id1 and id2, that contain the id numbers of these items.

171 Each row of this table describes an association. Furthermore, the projects package keeps

172 track of the order in which these associations appear so that the automatically generated

173 title pages list authors and affiliations in the correct order. Users are able to run functions

174 to reorder these associations as needed.

\section{Project File Structure}

176 Users create individual project folders with the function new_project( ). The name of each

177 project folder is of the form $\mathrm{p} X X X X$, where $X X X X$ is the project's id padded with 0s on the

178 left side. When a project folder is created, it is automatically populated with folders and

179 files as shown:

- $\quad / p X X X X$

181

- /data

182

- /data_raw

183

- /figures

184

- /manuscript

185

- /progs

- $\quad$ 01_protocol.Rmd 
191 The included subfolders serve to organize the project, while the. Rmd files are templates

192 that facilitate the user's workflow.

\section{File Management}

194 The goal of the projects package is to provide a comprehensive set of tools managing

195 project files in a way that is self-contained in $\mathrm{R}$ and independent of the underlying

196 operating system. On a daily basis, researchers make, move, copy, delete and archive files.

197 Through the projects package, researchers can perform all these actions in an organized

198 manner with an automated file structure. In fact, users are advised not to manipulate the

199 /projects folder and its content with their operating system, so that the package does not

200 lose track of these files. Multi-user application of projects requires a server or an

201 otherwise shared directory where multiple users can access the /projects folder. File-

202 managing functions-along with all functions-are demonstrated below (see

203 Demonstration).

\section{Other Features}

205 The projects package supports style customization of manuscripts through cascading

206 style sheets (CSS). When a project is created, a file called style.css is created alongside the

207 .Rmd files in the /progs folder; users can customize their protocol and report by editing this

208 file. Users can also create their own template files for the datawork, analysis and

209 manuscript.Rmd files. Lastly, the user is given the option to make these.Rmd files BibTeX-

210 ready for streamlined bibliography creation. 


\section{Demonstration}

212 Upon installation, the projects package must be set up using setup_projects( ). The user

213 is to input the file path of the directory wherein the /projects folder is desired to be located.

214 library(projects)

215 setup_projects("C:/")

216 \#\# "projects" folder created at

217 \#\# C:/projects

218 \#\#

219 \#\# Add affiliations with new_affiliation(), then add authors with

220 \#\# new_author(), then create projects with new_project()

221 As the message suggests, it is in the user's best interest to add affiliations, followed by

222 authors and projects.

new_affiliation(department_name = "Department of Physics", institution_name = "University of North Science", address $=$ "314 Newton Blvd, Springfield CT 06003")

\#\# New affiliation:

\#\# \# A tibble: $1 \times 4$

230 \#\# $1 \quad 1$ Department of Phys University of North S 314 Newton Blvd, Sprin

231 This affiliation has been successfully added to the "affiliations" table in the projects

232 relational database. The next code chunk creates a few more affiliations (output not

233 included).

new_affiliation(department_name = "Impossibles Investigation Team", institution_name $=$ "Creekshirebrook Academy of Thinks", address = "Let Gade 27182, 1566 Copenhagen, Denmark")

new_affiliation(department_name = "Statistical Consulting Unit", institution_name = "Creekshirebrook Academy of Thinks", address = "196 Normal Ave, Columbus, $\mathrm{OH} "$, id $=50$ )

240 Note that we chose a specific id number (50) for the affiliation called the "Impossibles

241 Investigation Team." 
242 Now we are ready to add authors to the "authors" table of the projects database.

243

244

245

246

247

248

249

250

251

252

253

254

255

256

257

258

259

260

261

262

263

264

265

266

267

268

269

270

271

272

273

274

275

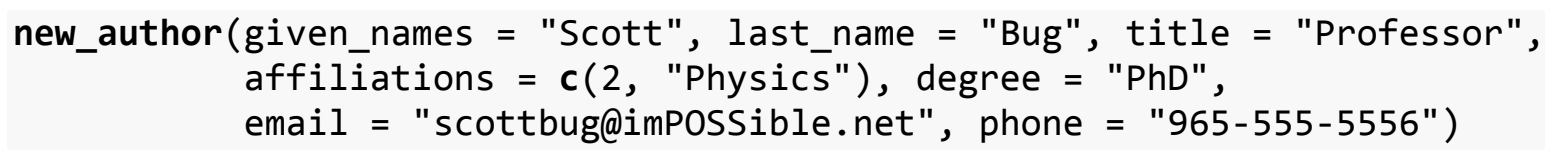

\#\# New author:

\#\# \# A tibble: 1 × 7

\#\# id given_names last_name title degree email phone

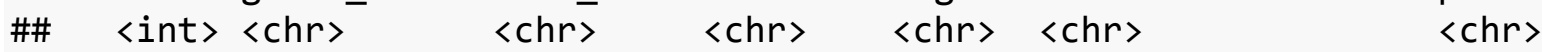

\#\# 11 Scott Bug Profess PhD scottbug@impossib 965-555-

\#\#

\#\# New author's affiliations:

\#\# \# A tibble: $2 \times 4$

\#\# affiliation_id department_name institution_name address

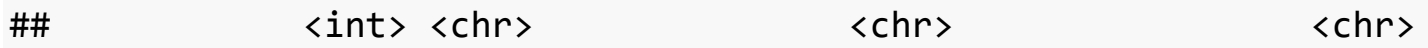

\#\# 12 Impossibles Invest Creekshirebrook Acad Let Gade 27182

\#\# 21 Department of Phys University of North 314 Newton Blv

In creating associations between Scott Bug and his affiliations, we were able to enter both

the id number of one of them (2) and a substring of the department_name of the other

("Physics"). The email address was coerced to lowercase.

Now we will add more authors (output not included).

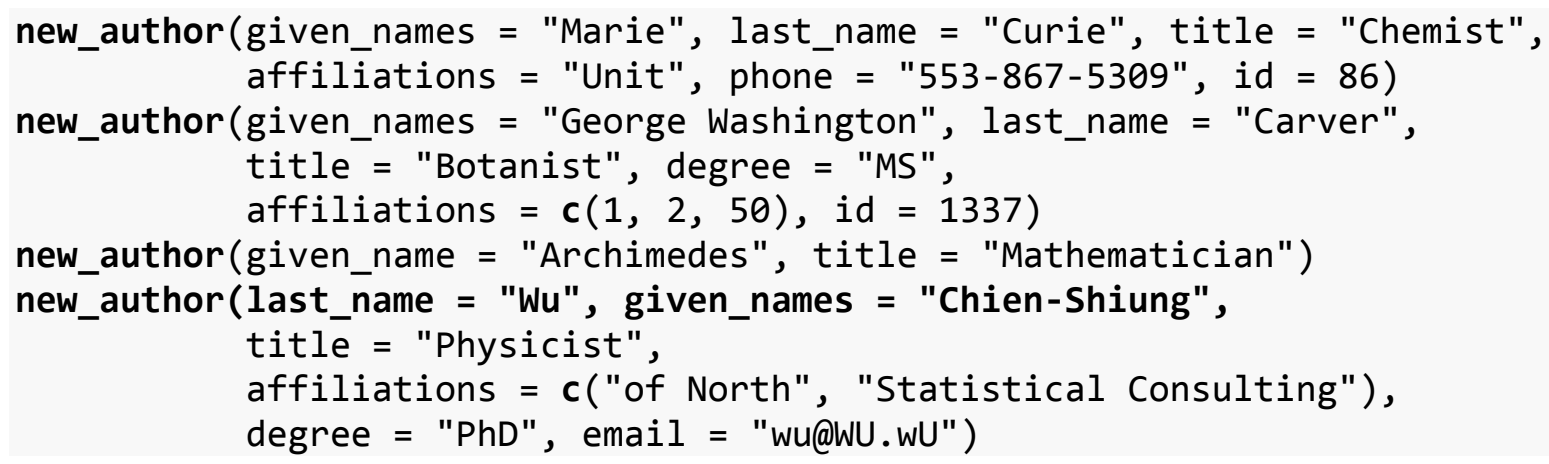

Now that some authors and affiliations have been created, we can view these tables:

authors()

\#\# \# A tibble: $5 \times 7$

\#\# id given_names last_name title degree email phone 

aCC-BY 4.0 International license.

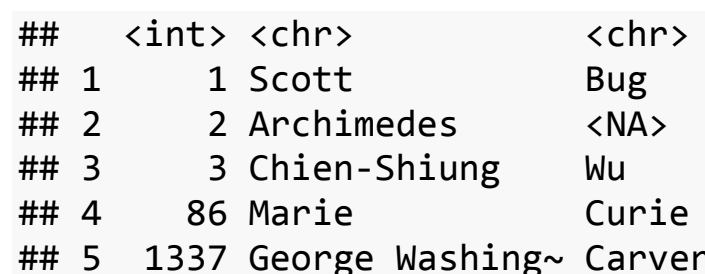

$\langle$ chr >

Bug

$\langle N A>$

Wu

Curie

279

280

281

282

283

284

285

286

287

288

289

290

291

292

293

294

295

296

297

298

299

300

301

302

303

304

305

306

307

308

309

310

311

312

313

314

315

\section{affiliations()}

\#\# \# A tibble: $3 \times 4$

\#\# id department_name 〈int> 〈chr>

Now we will showcase project creation: institution_name

$\langle$ chr>

$\begin{array}{llll}\text { 〈chr } & \langle\mathrm{chr}\rangle & \langle\mathrm{chr}\rangle & \langle\mathrm{ch} r\rangle \\ \text { Professor } \mathrm{PhD} & \text { scottbug@impo } & 965-555 \sim \\ \text { Mathemat } & \langle\mathrm{NA}\rangle & \langle\mathrm{NA}\rangle & \langle\mathrm{NA}\rangle \\ \text { Physicist } \mathrm{PhD} & \text { wu@wu.wu } & \langle\mathrm{NA}\rangle \\ \text { Chemist } & \langle\mathrm{NA}\rangle & \langle\mathrm{NA}\rangle & 553-867 \sim \\ \text { Botanist } \mathrm{MS} & \langle\mathrm{NA}\rangle & \langle\mathrm{NA}\rangle\end{array}$

\#\# 11 Department of Physics University of North Sc 314 Newton Blvd, Sp \#\# 22 Impossibles Investig Creekshirebrook Academ Let Gade 27182, 156 \#\# 350 Statistical Consulti Creekshirebrook Academ "196 Normal Ave, Co

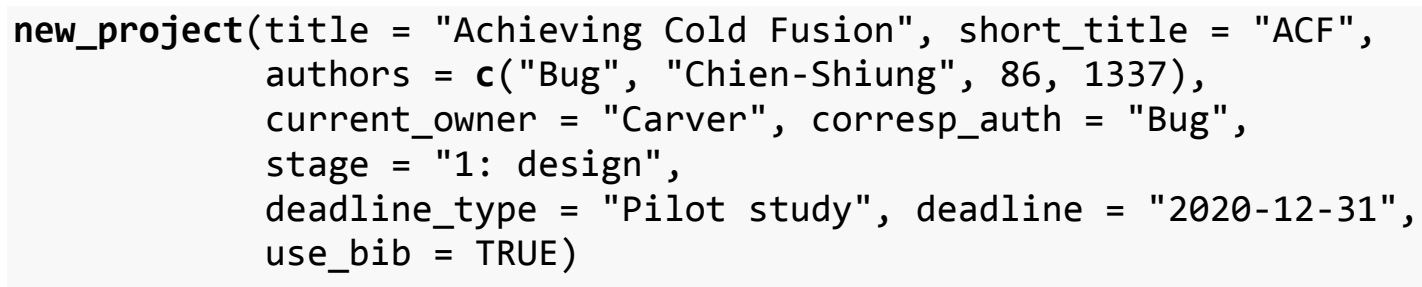




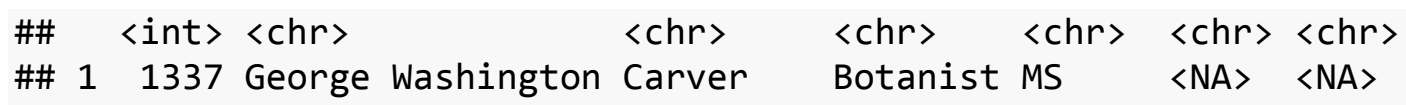

\#\#

319 \#\# Corresponding author:

320 \#\# \# A tibble: $1 \times 7$

\#\# 11 Scott $\quad$ Bug

Profess PhD

scottbug@impossib 965-555-

$324 \quad \# \#$

325 \#\# Creator: nkrieger

326 Since a creator was not specified, this field was populated with the value of

Sys.info()["user"].

328 Among other files and folders, this line of code created the files 01_protocol.Rmd and

329 04_report.Rmd, which both include code to create a title page exhibiting a preformatted list

330 of authors and their affiliations.

331 Here is what the top of these files look like:

$---$

333

title: "Achieving Cold Fusion"

output:

word_document: default

335

336

337

html_document:

css: style.css

338

bibliography: p0001.bib

339

340

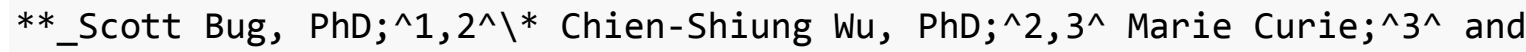
George Washington Carver, $\mathrm{MS}^{\wedge} 1,2,3^{\wedge}{ }_{-}^{* *}$

| ^1^ Impossibles Investigation Team, Creekshirebrook Academy of Thinks, Let Gade 27182, 1566 Copenhagen, Denmark I ${ }^{\wedge}{ }^{\wedge}$ Department of Physics, University of North Science, 314 Newton Blvd, Springfield CT 06003 I $\wedge 3^{\wedge}$ Statistical Consulting Unit, Creekshirebrook Academy of Thinks, 196 Normal Ave, Columbus, $\mathrm{OH}$ 


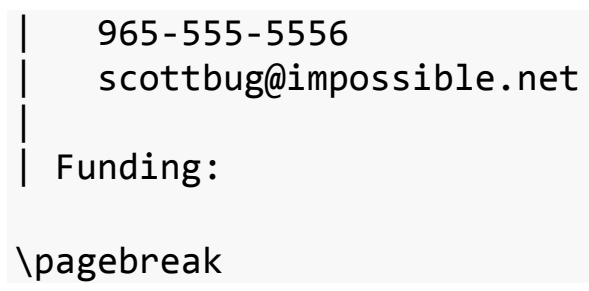

359 We notice that the author order given to the authors argument in new_project()

360 command has been preserved; furthermore, Scott Bug has been marked as the

361 corresponding author and his contact information has been included. Once this . Rmd file is

362 rendered, this will become a proper title page.

363 More projects can be created as follows:

364

365

366

367

368

369

370

371

372

373

374

375

376

377

378

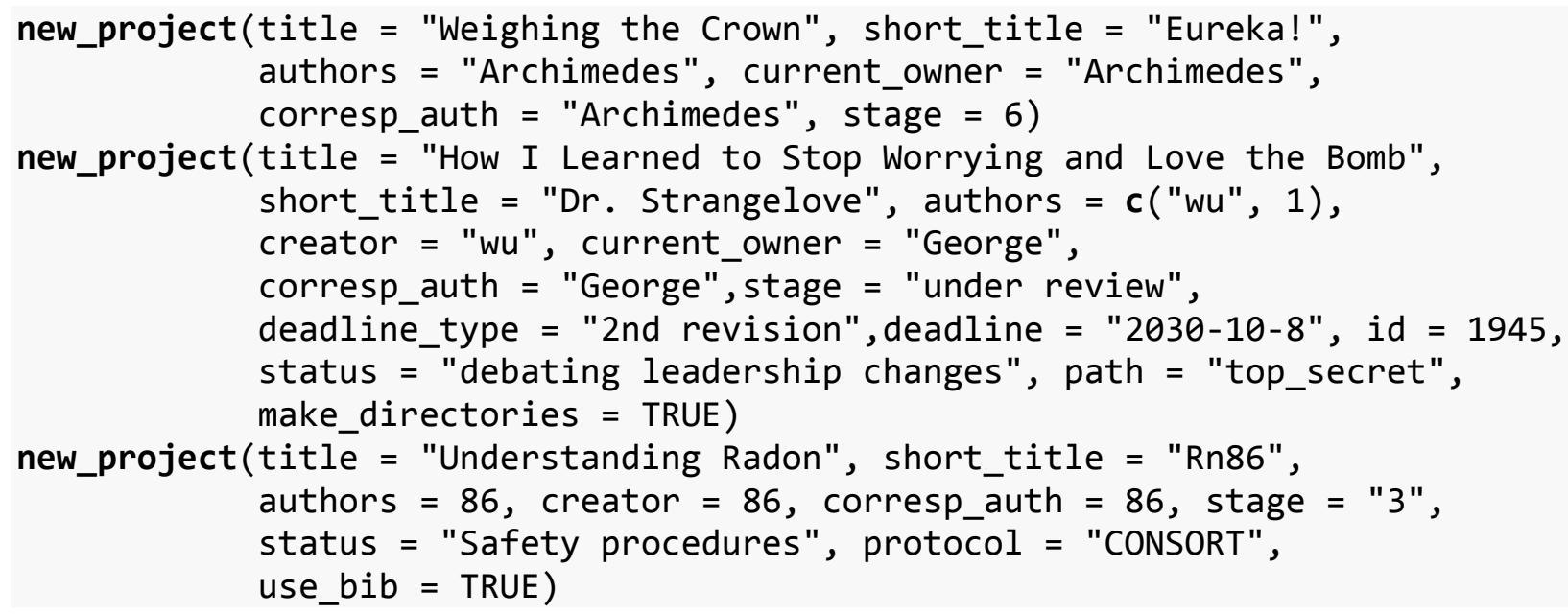

Below is the list of all projects that have been created:

\section{$\operatorname{projects}()$}

\#\# \# A tibble: $4 \times 11$

\#\# id title short_title current_owner status deadline_type deadline

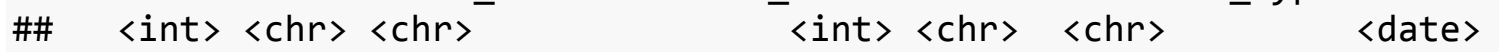

\#\# 11 Achi ACF 1337 just Pilot study 2020-12-31

\#\# $2 \quad 2$ Weig Eureka!

\#\# 33 Unde Rn86

2 just $\sim\langle N A\rangle$

\#\# 41945 How Dr. Strang 1337 debat 2nd revision 2030-10-08

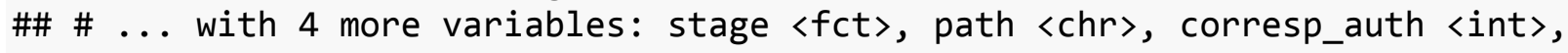
\#\# \# creator 〈chr > 
389 Projects, authors and affiliations can be edited with edit_project(), edit_author( ) and

390 edit_affiliation( ), respectively. For example, we can add affiliations to and remove

391 affiliations from an author with:

392 edit_author(author = "Bug", affiliations $=\sim+50$ - impossibles)

393 \#\# Edited author:

394 \#\# \# A tibble: $1 \times 7$

395 \#\# id given_names last_name title degree email phone

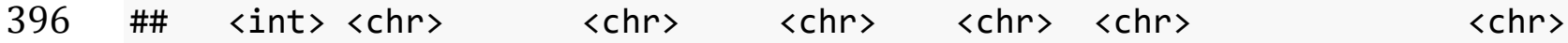

397 \#\# 1 1 Scott Bug Profess PhD scottbug@impossib 965-555-

398 \#\#

399 \#\# Edited author's affiliations:

400 \#\# \# A tibble: $2 \times 4$

401 \#\# affiliation_id department_name institution_name address

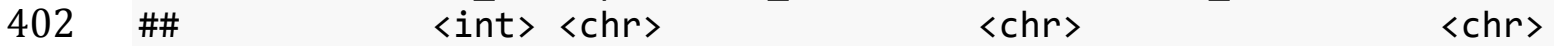

403 \#\# $1 \quad 1$ Department of Phys University of North 314 Newton Blv

404 \#\# $2 \quad 50$ Statistical Consul Creekshirebrook Acad "196 Normal Av

405 When adding or removing affiliations/authors from an author/project, a one-sided formula

406 is used: it must begin with a tilde ( ), and elements are added with + and removed with -.

407 Elements can be referred to by their id numbers or their names, as described above.

408 A formula is also used in the authors argument in edit_project( ):

edit_project("Cold", title = "Cold Fusion Is Actually Impossible", authors $=\sim$ "archi", stage = "accepted")

411 \#\# Edited project info:

412 \#\# \# A tibble: $1 \times 11$

413 \#\# id title short_title current_owner status deadline_type deadline

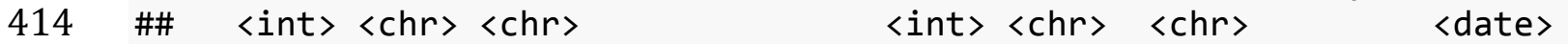

$415 \quad \# \# 1 \quad 1$ Cold ACF 1337 just Pilot study 2020-12-31

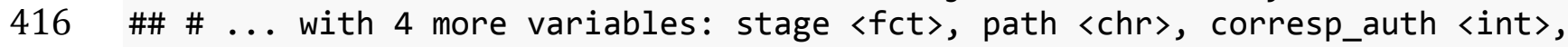

417 \#\# \# creator 〈chr>

$418 \quad \# \#$

419 \#\# Edited project's authors: 
\#\# \# A tibble: $5 \times 7$

421

\#\# author_id given_names

\#\#

\section{last_name title}

degree email

phone

422

423

424

425

426

427

428

429

430

431

432

433

434

435

436

437

438

439

440

441

442

443

444

445

446

447

\#\# 1

\#\# 2

\#\# 3

\#\# 4

\#\# 5

\#\#

\#\# title: "Cold Fusion Is Actually Impossible"

\#\#

\#\#

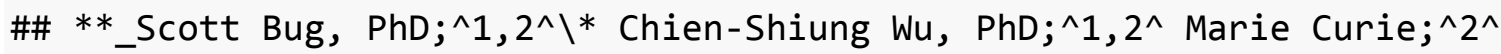

Archimedes; and George Washington Carver, $\mathrm{MS}^{\wedge} 1,2,3^{\wedge}{ }^{* *}$

\#\#

\#\# | $1^{\wedge}$ Department of Physics, University of North Science, 314 Newton Blvd, Springfield CT 06003

\#\# | ${ }^{\wedge}{ }^{\wedge}$ Statistical Consulting Unit, Creekshirebrook Academy of Thinks, 196

Normal Ave, Columbus, $\mathrm{OH}$

\#\# | ^3^ Impossibles Investigation Team, Creekshirebrook Academy of Thinks,

Let Gade 27182, 1566 Copenhagen, Denmark

\#\#

\#\# | \* Corresponding author

\#\# 314 Newton Blvd, Springfield CT 06003

\#\# 965-555-5556

\#\# | scottbug@impossible.net

\#\#

\#\# | Funding:

448 Here, the title and stage of the project have also been edited. The default behavior of

449 edit_project() is to reprint the project title as well as the other elements of the title

450 page. The user can then copy and paste this header into the 01_protocol.Rmd and

451 04_report.Rmd files.

452 We also notice that the default behavior when adding elements is to place them before the

453 last author (unless there was only one author). This occurs after elements are removed, as

454 specified by any minus signs (-) in the formula.

455 Another function that affects author order and whose default behavior reprints project title

456 page information is reorder_authors( ): 
458 \#\# project info:

459 \#\# \# A tibble: 1 x 11

460 \#\# id title short_title current_owner status deadline_type deadline

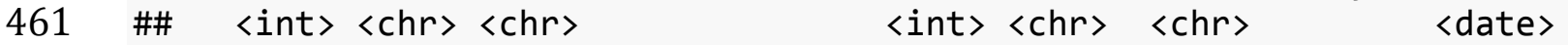

$462 \quad \# \# 1 \quad 1$ Cold ACF 1337 just $\sim$ Pilot study 2020-12-31

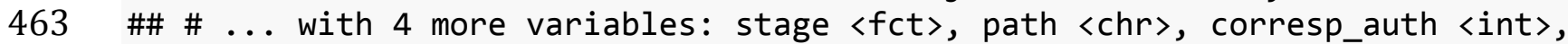

464 \#\# \# creator 〈chr>

$465 \quad \# \#$

466 \#\# Reordered project authors:

467 \#\# \# A tibble: $5 \times 7$

468 \#\# author_id given_names last_name title degree email phone

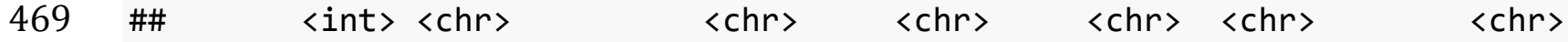

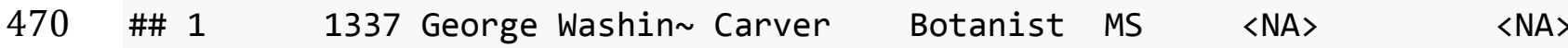

471 \#\# $2 \quad 1$ Scott Bug Professor PhD scottbug@im 965-55

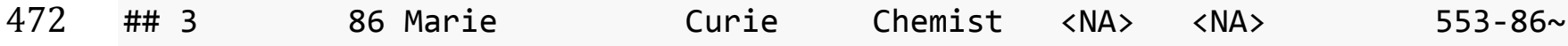

473 \#\# 4 Chien-Shiung Wu Physicist PhD wu@wu.wu $3 \mathrm{NA}\rangle$

474 \#\# 5 Archimedes $\quad$ NA $\quad$ Mathemat $\langle\mathrm{NA}\rangle \quad\langle\mathrm{NA}\rangle \quad\langle\mathrm{NA}\rangle$

$475 \quad \# \#$

476 \#\# title: "Cold Fusion Is Actually Impossible"

477 \#\#

$478 \quad \# \#$

479 \#\# **_George Washington Carver, MS;^1,2,3^ Scott Bug, PhD;^1, $3^{\wedge} \backslash^{*}$ Marie

480 Curie;^ $3^{\wedge}$ Chien-Shiung Wu, PhD;^1, $3^{\wedge}$ and Archimedes_**

481 \#\#

$482 \# \# \mid \wedge 1^{\wedge}$ Department of Physics, University of North Science, 314 Newton Blvd, 483 Springfield CT 06003

$484 \# \# \mid{ }^{\wedge} 2^{\wedge}$ Impossibles Investigation Team, Creekshirebrook Academy of Thinks,

485 Let Gade 27182, 1566 Copenhagen, Denmark

$486 \# \# \mid \wedge 3^{\wedge}$ Statistical Consulting Unit, Creekshirebrook Academy of Thinks, 196

487 Normal Ave, Columbus, $\mathrm{OH}$

488 \#\# |

$489 \# \#$ | $\backslash *$ Corresponding author

$490 \quad \# \# \mid 314$ Newton Blvd, Springfield CT 06003

$491 \quad \# \#$ 965-555-5556

492 \#\# | scottbug@impossible.net

$493 \quad \# \#$

$494 \# \# \mid$ Funding:

495 Importantly, edit_author(), edit_affiliation( ), and reorder_affiliations() do not

496 reprint the title pages of all affected projects. Fortunately, the user can reprint updated title

497 pages with the header ( ) function: 
edit_affiliation(affiliation= "Impossibles",

header $($ project $=$ "Cold")

title: "Cold Fusion Is Actually Impossible"

**_George Washington Carver, MS;^1,2, $3^{\wedge}$ Scott Bug, PhD;^1, $3^{\wedge} \backslash^{*}$ Marie Curie;^ $3^{\wedge}$ Chien-Shiung Wu, PhD;^1, $3^{\wedge}$ and Archimedes_**

| ^1^ Department of Physics, University of North Science, 314 Newton Blvd, Springfield CT 06003

I ^2^ Pseudoscience Debunking Unit, Creekshirebrook Academy of Thinks, Let Gade 27182, 1566 Copenhagen, Denmark I $\wedge^{\wedge}$ Statistical Consulting Unit, Creekshirebrook Academy of Thinks, 196 Normal Ave, Columbus, $\mathrm{OH}$

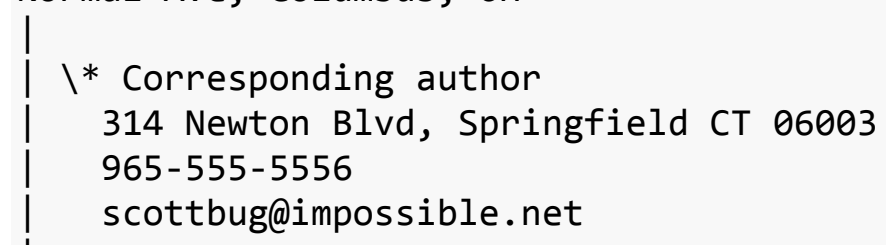

521 In order to organize projects, users can create subdirectories within the main /projects

522 folder where individual project folders can dwell. Among the examples above, this has

523 already occurred with the project with the nickname (i.e., short_title) "Dr. Strangelove"

524 because on its creation the arguments path $=$ top_secret and make_directories $=$ TRUE

525 were included. The latter argument must be TRUE if the desired path does not already exist.

526 Observe the path column among the existing projects:

527 projects() \% \% select(id, short_title, path)

528 \#\# \# A tibble: $4 \times 3$

529 \#\# id short_title path

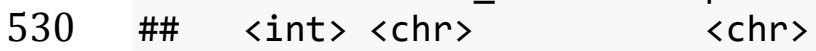

531 \#\# 1 1 ACF $\quad$ C:/projects/p0001

532 \#\#2 2 Eureka! C:/projects/p0002

533 \#\# $3 \quad 3$ Rn86 C:/projects/p0003

534 \#\# $41945 \mathrm{Dr}$. Strangelove C:/projects/top_secret/p1945

535 Users can also create subdirectories with the function new_project_group( ): 
new_project_group("Greek_studies/ancient_studies")

$537 \quad \# \#$

538 \#\# The following directory was created:

539 \#\# C:/projects/Greek_studies/ancient_studies

540 If a project has already been created, it can be moved not with edit_project() but with

541 move_project(). Users can also copy projects using copy_project(); everything in the

542 copy will be the same except its id, folder name (which, again, is based on its id), path

543 (which, again, is based on its folder name), and the name of its .Rproj file (which has the

544 same name as the folder).

545 move_project("Crown", path = "Greek_studies/ancient_studies")

546 copy_project(project_to_copy = "Radon",

547

548

549

550

551

552

553

554

555

$$
\begin{aligned}
& \text { path }=\text { "dangerous_studies/radioactive_studies/radon_studies", } \\
& \text { make_directories = TRUE) }
\end{aligned}
$$

556 Projects can also be archived; they are moved into a subdirectory called /archive that is at

557 the same level as the project folder $(/ p X X X X)$ before it was run. If this /archive folder does

558 not exist, it will be created.

archive_project("Strangelove")

560

\#\# \# A tibble: $1 \times 11$

561

\#\# id title short

562

\#\# <int $\rangle\langle\mathrm{chr}\rangle\langle\mathrm{chr}\rangle$

path

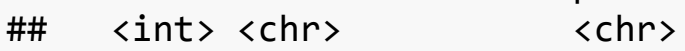

\#\# 12 Weighing the C C:/projects/Greek_studies/ancient_studies/p0002

\#\# 23 Understanding $\sim \mathrm{C}: /$ projects/p0003

\#\# 34 Understanding C:/projects/dangerous_studies/radioactive_studies

563

564

565

\#\# 11945 How Dr. Strang 1337 debat 2nd revision 2030-10-08

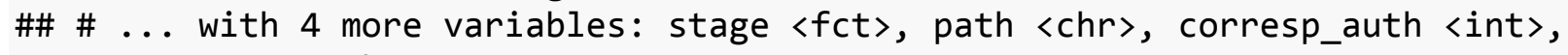
\#\# \# creator 〈chr>

566 \#\#

567 \#\# The above project was archived and has the file path

568 \#\# C:/projects/top_secret/archive/p1945 
569 When a project is archived, it is no longer included in projects() output unless the user

570 sets archived $=$ TRUE.

571 projects() \%> select(id, short_title, path)

572 \#\# \# A tibble: $4 \times 3$

573 \#\# id short_title path

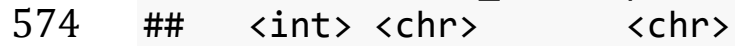

$575 \quad \# 1 \quad 1$ ACF 1 C:/projects/p0001

576 \#\# 2 Eureka! C:/projects/Greek_studies/ancient_studies/p0002

577 \#\# 3 Rn86 C:/projects/p0003

578 \#\# $4 \quad 4$ Rn86 C:/projects/dangerous_studies/radioactive_studies/rad

579 projects (archived = TRUE) \%> select (id, short_title, path)

580 \#\# \# A tibble: $5 \times 3$

581 \#\# id short_title path

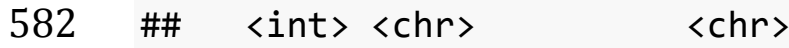

$583 \quad \# \# 1 \quad 1 \mathrm{ACF} \quad$ C:/projects/p0001

584 \#\# 2 Eureka! C:/projects/Greek_studies/ancient_studies/p0002

585 \#\# $3 \quad 3$ Rn86 C:/projects/p0003

586 \#\# $4 \quad 4$ Rn86 C:/projects/dangerous_studies/radioactive_studies/

587 \#\# 51945 Dr. Strangelo C:/projects/top_secret/archive/p1945

588 Lastly, affiliations, authors and projects can be deleted with delete_affiliation(),

589 delete_author( ) and delete_project( ), respectively. Deleting an author is complete:

590 doing so removes the author from the creator, current_owner and corresp_auth fields of

591 all projects. Furthermore, deleting a project also deletes the entire project folder. Users

592 should use the delete functions with caution.

593 delete_affiliation("north science")

594 \#\# \# A tibble: $1 \times 4$

595 \#\# id department_name institution_name address

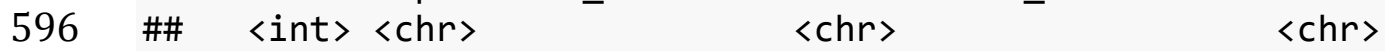

$597 \quad \# \# 1 \quad 1$ Department of Phys University of North S 314 Newton Blvd, Sprin

598 \#\# \# A tibble: 1 x 4

599 \#\# id department_name institution_name address

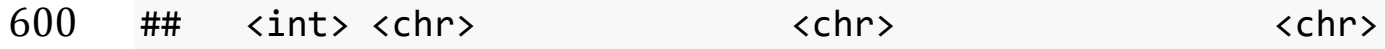

601 \#\# $1 \quad 1$ Department of Phys University of North S 314 Newton Blvd, Sprin

602 \#\# The above affiliation was deleted.

603 delete_author(2) 
604 \#\# \# A tibble: $1 \times 7$

605

\#\# id given names

606

\#\#

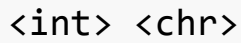

last_name title

degree email phone

607

\#\# 12 Archimedes

$\langle c h r\rangle \quad\langle c h r\rangle$

$\langle c h r\rangle\langle c h r\rangle\langle c h r\rangle$

608

\#\# \# A tibble: $1 \times 7$

609

\#\#

id given_names

$\langle N A\rangle$

Mathematician 〈NA〉

$\langle\mathrm{NA}\rangle\langle\mathrm{NA}\rangle$

610

\#\#

<int> 〈chr>

last_name title

degree email phone

611

\#\# 1

2 Archimedes

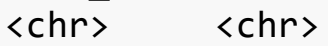

$\langle\mathrm{chr}\rangle\langle\mathrm{chr}\rangle\langle\mathrm{chr}\rangle$

612 \#\# The above author was deleted.

613 delete_project("Crown")

614 \#\# \# A tibble: $1 \times 11$

615

616

\#\# id title short_title

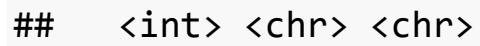

\#\# 12 Weig Eureka!

Mathematician 〈NA〉

$\langle\mathrm{NA}\rangle\langle\mathrm{NA}\rangle$

617

618

619

620

621

622

623

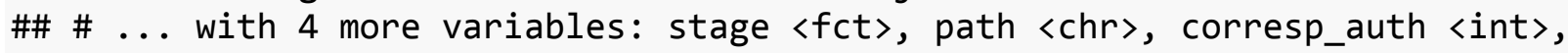

\#\# \# creator 〈chr>

\#\# \# A tibble: $1 \times 11$

\#\# id title short_title current_owner status deadline_type deadline

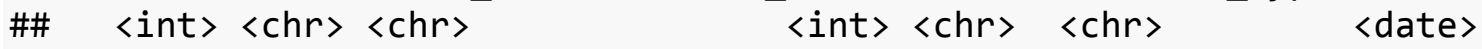

\#\# $1 \quad 2$ Weig Eureka!

NA just $~\langle N A\rangle$

NA

624

625

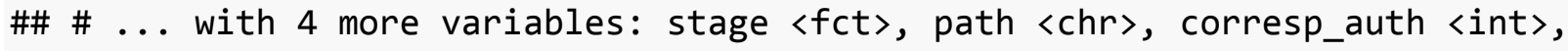
\#\# \# creator 〈chr >

626 \#\#

627 \#\# The above project was deleted.

628 Discussion

629 The projects package provides a comprehensive set of tools for reproducible team science

630 workflows. Efficiency in project management, including manuscript development, is

631 facilitated by an internal database that keeps record of project details as well as team

632 members' affiliations and contact information. For manuscripts, title pages are

633 automatically generated from this database, and a selection of manuscript outlines

634 compliant with reporting guidelines are available upon installation. Other workflow-

635 related R packages exist, such as workflowr, which offers collaborative project file

636 management on Github. The projects package, however, is unique in its focus on 
637 manuscript development within the typical academic research setting where data are

638 firewalled and research teams collaborate within secure networks. (5)

639 The projects package builds upon existing tools that facilitate reproducibility in small

640 ways. R Markdown, for example, allows reviewers to intuitively investigate R scripts piece

641 by piece in order to inspect the integrity of data management and to validate intermediate

642 results; however, the R Markdown file format does not by itself organize project files. In

643 addition, the here package makes file paths generalizable so that networks of R scripts will

644 run without error on multiple computers with different parent file structures; however, it

645 does not standardize file names and paths in the project's immediate working directory.

646 The projects package not only adopts the benefits of R Markdown and here in its

647 templates but also facilitates file management with a standardized file structure. Thus, the

648 user's network of R scripts and data objects maintains continuity. Collaborators and

649 reviewers can-at least in principle-run a projects user's entire workflow on their own

650 computers without file-path-related errors. This streamlines both replication attempts as

651 well as the investigation of reproducibility.

652 The main limitation of the projects package lies in its setting: it is confined to the $\mathrm{R}$

653 statistical programming language, which not all researchers know and use. Prospective

654 users of the package who do not know R must spend time learning how to use it, and this

655 drawback is compounded as the size of the transitioning research team increases.

656 Researchers who do know R may need to spend time gaining proficiency with R Markdown

657 and other projects package dependencies. In future work, we will explore ways to 
658 integrate the functionality of the projects package with other statistical programming

659 languages (e.g., Python and SAS).

660 In spite of the inevitable learning curve that is present when adapting to a new

661 programming language, the projects package is intuitive to use among regular users of $\mathrm{R}$

662 and R Markdown. We believe that the projects package may be useful for teams that

663 manage multiple collaborative research projects in various stages of development. It has

664 the potential to improve both the quality and efficiency of individual and team researchers

665 while also rendering the task of maintaining reproducibility less cumbersome. The open-

666 source nature of the R environment ensures that the projects package will only improve

667 with time and use, as the scientific community continues to embrace the tools essential for

668 maintaining a reproducible workflow.

\section{Acknowledgements}

670 The authors of this package acknowledge the support provided by members of the

671 Northeast Ohio Cohort for Atherosclerotic Risk Estimation (NEOCARE) investigative team:

672 Claudia Coulton, Douglas Gunzler, Darcy Freedman, Neal Dawson, Michael Rothberg, David

673 Zidar, David Kaelber, Douglas Einstadter, Alex Milinovich, Monica Webb Hooper, Kristen

674 Hassmiller-Lich, Ye Tian (Devin), Kristen Berg, and Sandy Andrukat. 


\section{References}

677 1. Gilbert DT, King G, Pettigrew S, Wilson TD. Comment on Estimating the reproducibility of 678 psychological science. Science [Internet]. 2016;351(6277):1037-7. Available from:

679 http://science.sciencemag.org/content/351/6277/1037.2

680 2. The Open Science Collaboration. Estimating the reproducibility of psychological science.

681 Science [Internet]. 2015;349(6251). Available from:

682 http://science.sciencemag.org/content/349/6251/aac4716

683 3. Nosek BA, Alter G, Banks GC, Borsboom D, Bowman SD, Breckler SJ, et al. Promoting an

684 open research culture. Science [Internet]. 2015;348(6242):1422-5. Available from:

685 http://science.sciencemag.org/content/348/6242/1422

686 4. Baker M. 1,500 scientists lift the lid on reproducibility. Nature News.

$6872016 ; 533(7604): 452$.

688 5. Blischak J, Carbonetto P, Stephens M. Workflowr: A framework for reproducible and

689 collaborative data science [Internet]. 2018. Available from: https://CRAN.R-

690 project.org/package=workflowr 


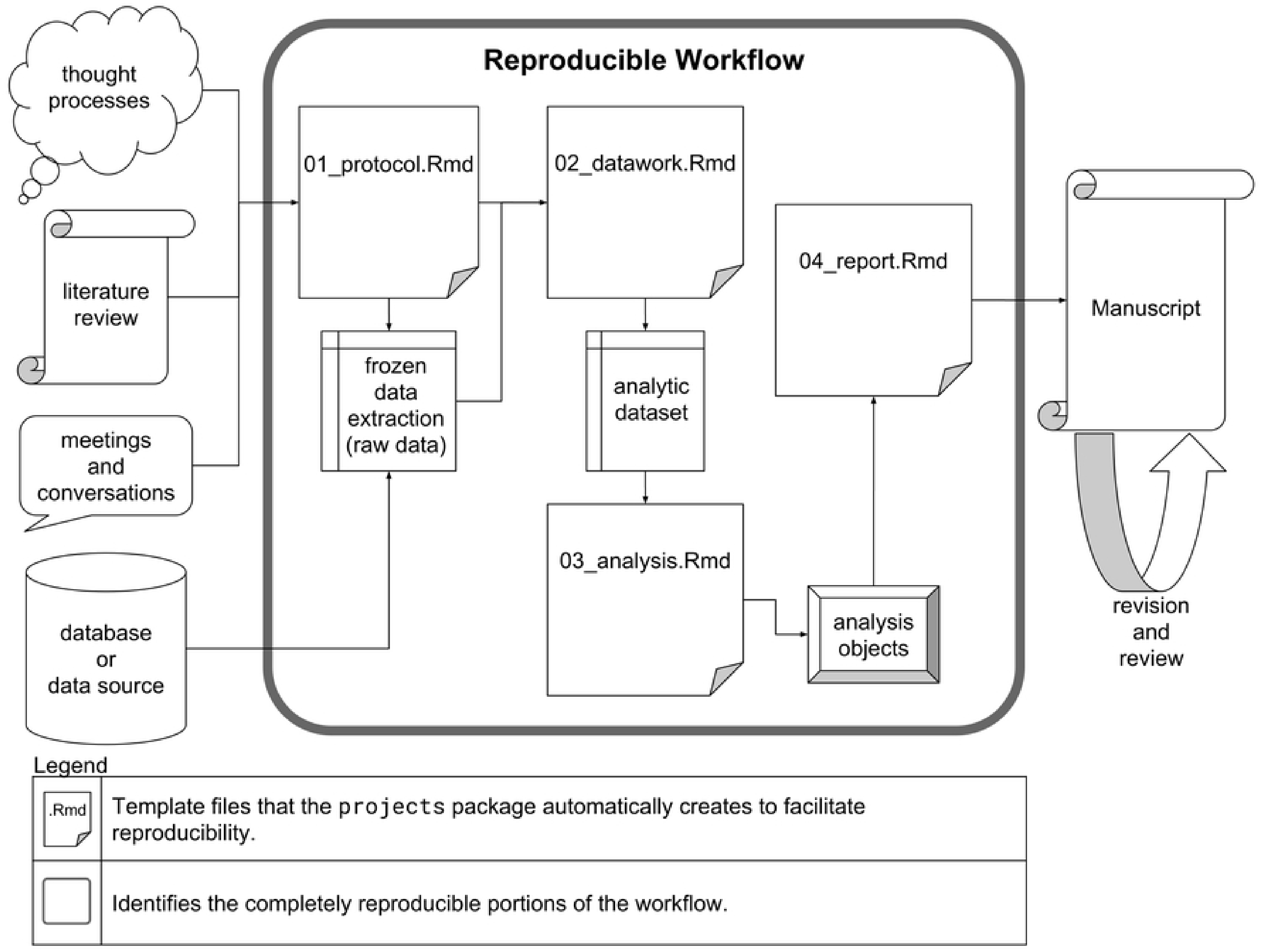

Fig 1 